\title{
Existence of Necessary Condition for Normal Solution Operator Equation
}

\author{
${ }^{1}$ Md Najmul Hoda*, ${ }^{2}$ Mohammad Abid Ansari \\ ${ }^{1}$ Research Scholar* Department of Mathematics, T.M. Bhagalpur University, Bhagalpur-812007, India \\ ${ }^{2}$ Associate Professor in Mathematics T.N.B. College, Bhagalpur, T.M. Bhagalpur University, Bhagalpur- \\ 812007, India \\ E-mail:najmul85hoda@gmail.com \\ Corresponding author*
}

Abstract: An operator means a bounded linear operator on Hilbert span. This paper proves the assertion made in its title. Following theorem yields the famous result

$$
A B+B A^{*}=I=A^{*} B+B A
$$

Where $A$ and $B$ are the bounded linear operator on a Hilbert span $H$. where $B^{*}$ is self adjoint satisfying the above equation. After modification of this equation some interesting results are obtained.

Theorem: If $\mathrm{AB}+\mathrm{BA}^{*}=\mathrm{I}$ has solution $\mathrm{B}$, then $0 \in \sigma_{\delta}(\mathrm{A})$ and $0 \in \rho(B)$. Further $\left\|B^{-1}\right\| \leq 2\|A\|$. and $0 \in \rho(\operatorname{Re} B)$.

Proof. If $\sigma_{\pi}$ (A) denotes the approximate point spectrum of A then $\sigma_{\pi}\left(\mathrm{A}^{*}\right)=\overline{\sigma_{\delta}(A)}$ bar is complex conjugate. Let $0 \in \sigma_{\delta}(A)$, then $0 \in \sigma_{\pi}\left(A^{*}\right)$, and so there exist a sequence $\left\{X_{n}\right\}$ of unit vector in $\mathrm{H}$ such that $A^{*} x_{n} \rightarrow 0$ as $n \rightarrow \infty$.

Now $\mathrm{AB}+\mathrm{BA}^{*}=\mathrm{I}$

$\Rightarrow \quad 1=\left(x_{n}, x_{n}\right)=\left(\left(A B+B A^{*}\right) x_{n}, x_{n}\right)$

or, $\quad 1=\left(A B x_{n}, x_{n}\right)+\left(B A^{*} x_{n}, x_{n}\right)$

$=\left(B x_{n}, A^{*} x_{n}\right)+\left(A^{*} x_{n}, B x_{n}\right) \rightarrow 0$

as $\quad n \rightarrow \infty$.

This is contradiction, hence $0 \notin \sigma_{\delta}(A)$.

Similarly we can prove that $0 \notin \sigma_{\delta}(B)$. since B is a self adjoint, we have $\sigma_{\delta}(B)=\sigma(B)$ Hence $0 \in \rho(B)$

Next we suppose that $x \in \mathrm{H}$ be arbitrary then

$$
\begin{array}{ll} 
& \|x\|^{2}=\left(\left(A B+B A^{*}\right) x, x\right) \\
= & (A B x, x)+\left(B A^{*} x, x\right) \\
= & \left(B x, A^{*} x\right)+\left(A^{*} x, B x\right) \\
& \leq\|B x\|\left\|A^{*} x\right\|+\left\|A^{*} x\right\|\|B x\| \\
= & 2\|B x\|\|A x\| \\
\text { i.e. } & 1 \leq 2\|B\|\|A\|
\end{array}
$$


i.e. $\quad\left\|B^{-1}\right\| \leq 2\|A\|$

Now we suppose that $0 \in \sigma_{\delta}(\operatorname{Re} \mathrm{A})$, this means that $0 \in \sigma_{\pi}(\operatorname{Re} \mathrm{A})$, hence there exists a sequence $\left\{x_{n}\right\}$ of unit vectors in $\mathrm{H}$ such that $\operatorname{Re}(A) x_{n} \rightarrow 0$ as $n \rightarrow \infty$ we have

$$
\begin{array}{cc} 
& 2 I=A B+B A^{*}+A^{*} B+B A \\
\Rightarrow & 2\left(x_{n}, x_{n}\right)=\left(\left(A+A^{*}\right) B+B\left(A+A^{*}\right) x_{n}, x_{n}\right) \\
\text { or, } & 2=2\left[\left(\operatorname{Re} A B x_{n}, x_{n}\right)+\left(B \operatorname{Re} A x_{n}, x_{n}\right)\right] \\
\text { or, } & I=\left(B x_{n}, \operatorname{Re} A x_{n}\right)+\left(\operatorname{Re} A x_{n}, B x_{n}\right) \rightarrow 0 \\
\text { as } & n \rightarrow \infty
\end{array}
$$

This is contradiction, hence $0 \notin \sigma_{\delta}(\operatorname{Re} \mathrm{A})$. Since $(\operatorname{Re} \mathrm{A})$ is self adjoint, hence $\sigma_{\delta}(\operatorname{Re} \mathrm{A})=\sigma(\operatorname{Re} \mathrm{A})$.

$\Rightarrow \quad 0 \in \rho(\operatorname{Re} A)$

Theorem : If (i) has a solution $B>0$ (i.e. $(B x, x)>0$ for all $x \in H /\{0\})$. Then there is an inner product on $H$, equivalent to the inner product (.,.), such that $\operatorname{Re} A>0$ with respect to it.

Proof. We define a new equivalent inner product on $\mathrm{H}$ by

$$
\langle x, y\rangle=(x, B y), \text { for all } x, y \in H
$$

Since

$$
\left[B, A-A^{*}\right]=0 \text { we have }
$$

$$
B\left(A-A^{*}\right)=\left(A-A^{*}\right) B
$$

$\Rightarrow \quad\left(B\left(A-A^{*}\right) x, x\right)=\left(\left(A-A^{*}\right) B x, x\right)$

or, $\quad\left(\left(A-A^{*}\right) x, B x\right)=\left(B x,\left(A^{*}-A\right) x\right)$

$$
=\overline{\left(\left(A^{*}-A\right) x, B x\right)}
$$

or, $\quad\left\langle\left(A-A^{*}\right) x, x\right\rangle=\left\langle\overline{\left(A^{*}-A\right) x, x}\right\rangle$

or, $\langle A x, x\rangle-\left\langle A^{*} x, x\right\rangle=\overline{\left\langle A^{*} x, x\right\rangle}-\overline{\langle A x, x\rangle}$

or, $\langle A x, x\rangle+\overline{\langle A x, x\rangle}=\left\langle A^{*} x, x\right\rangle+\overline{\left\langle A^{*} x, x\right\rangle}$

or, $\operatorname{Re}\langle A x, x\rangle=\operatorname{Re}\left\langle A^{*} x, x\right\rangle$

Again from the equation (1) we have

$$
\begin{aligned}
2 I & =\left(A+A^{*}\right) B+B\left(A+A^{*}\right) \text { then for any } x \in H . \\
2(x, x) & =\left(\left(A+A^{*}\right) B x, x\right)+\left(B\left(A+A^{*}\right) x, x\right) \\
\text { Or, } \quad 2\|x\|^{2} & =\frac{\left(B x,\left(A^{*}+A\right) x\right)+\left(\left(A+A^{*}\right) x, B x\right)}{\left(\left(A^{*}+A\right) x, B x\right)}+\left(\left(A+A^{*}\right) x, B x\right) \\
& =\overline{\left\langle\left(A+A^{*}\right) x, x\right\rangle}+\left\langle\left(A+A^{*}\right) x, x\right\rangle
\end{aligned}
$$




$$
\begin{aligned}
& =\langle\overline{A x+x}\rangle+\left\langle A^{*} x, x\right\rangle+\langle A x, x\rangle+\left\langle A^{*} x, x\right\rangle \\
& =\langle A x, x\rangle+\langle\overline{A x, x}\rangle+\left\langle A^{*} x, x\right\rangle+\left\langle\overline{A^{*} x+x}\right\rangle \\
& =2 \operatorname{Re}\langle A x, x\rangle+2 \operatorname{Re}\left\langle A^{*} x, x\right\rangle \\
& =2 \operatorname{Re}\langle A x, x\rangle+2 \operatorname{Re}\langle A x, x\rangle \quad[\operatorname{By}(2)] \\
& =4 \operatorname{Re}\langle A x, x\rangle
\end{aligned}
$$

Or, $\quad \operatorname{Re}\langle A x, x\rangle=\frac{1}{2}\|x\|^{2}>0$ for all $x \in H$

Hence $\operatorname{Re} A>0$

Theorem : If there exists a solutions A to (1) such that the eigen vectors of $A^{*}$ span $H$, then $W(B) \subseteq R /\{0\}$.

Proof. Let $\lambda \in \sigma_{P}\left(A^{*}\right)$ (* the point spectrum of $\mathrm{A}^{*}$ ) and let $x \in H, x \notin 0$ be an eigen vector corresponding to $\lambda$. So we have $A^{*} x=\lambda x$ for all $x \in H$.

From (1) we have

$$
\begin{array}{lll} 
& 0=\left(\left(A-A^{*}\right) B x, x\right)+\left(B\left(A^{*}-A\right) x, x\right) \\
\text { or, } & 0=\left(B x,\left(A^{*}-A\right) x\right)+\left(\left(A^{*}-A\right) x, B x\right) \\
\text { or, } & 0=(B x, \lambda x-A x)+(\lambda x-A x, B x) \\
\text { or, } & 0=(B x, \lambda x)-(B x, A x)+(\lambda x, B x)-(A x, B x) \\
\text { or, } & 0=\bar{\lambda}(B x, x)-(B x, A x)+\lambda(B x, x)-(A x, B x) \\
\text { or, } & 0=(B x, A x)+(\overline{B x, A x})+(\lambda+\bar{\lambda})(B x, x) \\
\text { Or, } & \operatorname{Re}(B x, A x)=(\operatorname{Re} \lambda)(B x, x)
\end{array}
$$

Again we have

$$
\begin{aligned}
2\|x\|^{2} & =\left(\left(A+A^{*}\right) B x, x\right)+\left(B\left(A^{*}+A\right) x, x\right) \\
& =\left(B x,\left(A^{*}+A\right) x\right)+\left(\left(A^{*}+A\right) x, B x\right) \\
& =(B x, \lambda x+A x)+(\lambda x+A x, B x) \\
& =(B x, \lambda x)+(B x, A x)+(\lambda x, B x)+(A x, B x) \\
& =\bar{\lambda}(B x, x)+\lambda(B x, x)+(B x, A x)+(\overline{B x, A x}) \\
& =(\lambda+\bar{\lambda})(B x, x)+2 \operatorname{Re}(B x, A x) \\
& =2[\operatorname{Re} \lambda(B x, x)+\operatorname{Re} \lambda(B x, x)]
\end{aligned}
$$




$$
\begin{array}{ll}
\text { or, } 2\|x\|^{2} & =4(\operatorname{Re} \lambda)(B x, x) \\
\text { or, } 1 & =2(\operatorname{Re} \lambda)(B y, y) \quad \text { where } y=\frac{x}{\|x\|}
\end{array}
$$

So that $\|y\|=1$. Clearly $\quad \operatorname{Re} \lambda \neq 0$

This implies that

Hence

$$
(B y, y)=\frac{1}{2(\operatorname{Re} \lambda)} \text { for all } y \in H
$$

Theorem: If there exists a solution A to (1) such that the eigen vectors of A span $\mathrm{H}$, then $W(B) \subseteq R /\{0\}$.

Proof. Let $\lambda \in \sigma_{P}(A)$ and let $0 \notin x \in H$ be an eigen vector corresponding to $\lambda$. Then we have $A x=x$ for all $x \in H$

From equation (1) we have

$$
\begin{aligned}
& 0=\left(\left(A-A^{*}\right) B x, x\right)+\left(B\left(A^{*}-A\right) x, x\right) \\
&=\left(B x,\left(A^{*}-A\right) x\right)+\left(\left(A^{*}-A\right) x, B x\right) \\
&=\left(B x, A^{*} x-\lambda x\right)+\left(A^{*} x-\lambda x, B x\right) \\
&=\left(B x, A^{*} x\right)-\bar{\lambda}(B x, x)+\left(A^{*} x, B x\right)-\lambda(x, B x) \\
&=\left(B x, A^{*} x\right)+\left(\overline{B x, A^{*} x}\right)+\bar{\lambda}(B x, x)-\lambda(B x, x) \\
& \text { or, }(\lambda+\bar{\lambda})(B x, x)=\left(B x, A^{*} x\right)+\left(\overline{B x, A^{*} x}\right) \\
& \text { Or, } \quad \operatorname{Re} \lambda(B x, x)=\operatorname{Re}\left(B x, A^{*} x\right)
\end{aligned}
$$

Again we have

$$
\begin{aligned}
\text { or, } 2\|x\|^{2} & =\left(\left(A+A^{*}\right) B x, x\right)+\left(B\left(A^{*}+A\right) x, x\right) \\
\text { or, } 2\|x\|^{2} & =\left(B x,\left(A^{*}+A\right) x\right)+\left(\left(A^{*}+A\right) x, B x\right) \\
& =\left(B x, A^{*} x+\lambda x\right)+\left(A^{*} x+\lambda x, B x\right) \\
& =\left(B x, A^{*} x\right)+\bar{\lambda}(B x, x)+\left(A^{*} x, B x\right)+\lambda(x, B x) \\
& =(\lambda+\bar{\lambda})(B x, x)+\left(B x, A^{*} x\right)+\left(\overline{B x, A^{*} x}\right) \\
& =2 \operatorname{Re} \lambda(B x, x)+2 \operatorname{Re}\left(B x, A^{*} x\right) \\
\text { or, } 2\|x\|^{2} & =2[\operatorname{Re} \lambda(B x, x)+\operatorname{Re} \lambda(B x, x)] \quad \text { by }(4)
\end{aligned}
$$




$$
\begin{aligned}
& \text { or, } 2\|x\|^{2}=4 \operatorname{Re} \lambda(B x, x) \\
& \text { or, } 1 \quad=2 \operatorname{Re} \lambda(B y, y) \quad \text { where } y=\frac{x}{\|x\|} \\
& \Rightarrow(B y, y)=\frac{1}{2 \operatorname{Re} \lambda},(\operatorname{Re} \lambda \neq 0)
\end{aligned}
$$

This show that $W(B) \subseteq R /\{0\}$.

Theorem : If there exists a solution $\mathrm{B}$ to equation (1) such that the eigen vectors of $\mathrm{B}$ span $\mathrm{H}$, then either $W(A) \subseteq R /\{0\}$ or $W(\operatorname{Re} A) \subseteq R /\{0\}$.

Proof. Let $\lambda \in \sigma_{P}(B)$ and let $0 \neq x \in H$ be an eigen vector corresponding to $\lambda$. Then $B x=\lambda x$ for all $x \in H$.

From equation is (1) we have

$$
\begin{aligned}
0 & =\left(\left(A-A^{*}\right) B x, x\right)+\left(B\left(A^{*}-A\right) x, x\right) \\
& =\left(B x,\left(A^{*}-A\right) x\right)+\left(\left(A^{*}-A\right) x, B x\right) \\
& =\left(\lambda x,\left(A^{*}-A\right) x\right)+\left(\left(A^{*}-A\right) x, \lambda x\right) \\
& =\lambda\left[\left(x, A^{*} x\right)-(x, A x)\right]+\bar{\lambda}\left[\left(A^{*} x, x\right)-(A x, x)\right] \\
& =\lambda[(A x, x)-(\overline{A x, x})]-\bar{\lambda}[(A x, x)-(\overline{A x, x})] \\
& =(\lambda-\bar{\lambda})[(A x, x)-(\overline{A x, x})] \\
& =\left(2 \mathrm{I}_{\mathrm{m}} \lambda\right)\left(2 \mathrm{I}_{\mathrm{m}}(A x, x)\right) \\
& =4\left(\mathrm{I}_{\mathrm{m}} \lambda\right)\left(\mathrm{I}_{\mathrm{m}}(A x, x)\right) \\
& \Rightarrow \text { Either } \mathrm{I}_{\mathrm{m}}(A x, x)=0
\end{aligned}
$$

In this case $(A x, x)=(\overline{A x, x})$

Or $\quad \mathrm{I}_{\mathrm{m}} \lambda=0 \mathrm{In}$ this case $\lambda=\bar{\lambda}$

Again from (1) we have

$$
\begin{aligned}
2\|x\|^{2} & =\left(\left(A+A^{*}\right) B x, x\right)+\left(B\left(A+A^{*}\right) x, x\right) \\
& =\left(B x,\left(A^{*}+A\right) x\right)+\left(\left(A^{*}+A\right) x, B x\right) \\
& =\left(\lambda x,\left(A^{*}+A\right) x\right)+\left(\left(A^{*}+A\right) x, \lambda x\right) \\
& =\lambda\left[\left(x, A^{*} x\right)+(x, A x)\right]+\bar{\lambda}\left[\left(A^{*} x, x\right)+(A x, x)\right] \\
& =\lambda[(A x, x)+(\overline{A x+x})]+\bar{\lambda}[(A x, x)+(\overline{A x+x})]
\end{aligned}
$$




$$
=(\lambda+\bar{\lambda})[(A x, x)+(\overline{A x, x})]
$$

Now two cases arise

Case I : When $(A x, x)=(\overline{A x, x})$ then by (7)

We have

$$
\begin{aligned}
2\|x\|^{2} & =4(\operatorname{Re} \lambda)(A x, x) \\
\Rightarrow \quad l \quad & =2(\operatorname{Re} \lambda)(A y, y) \quad \text { where } y=\frac{x}{\|x\|}
\end{aligned}
$$

Obviously by $\operatorname{Re} \lambda \neq 0$. This implies that

$(A y, y)=\frac{1}{2(\operatorname{Re} \lambda)}$ for all $y \in H$.

Hence $W(A) \subseteq R /\{0\}$.

Case II : $\quad$ When $\lambda=\bar{\lambda}$; i.e. $\lambda$ is purely real from equation (7) we have

$$
\begin{aligned}
2\|x\|^{2} & =2 \lambda[(A x, x)+(\overline{A x, x})] \\
& =2 \lambda 2 \operatorname{Re}(A x, x) \\
& =4 \lambda \operatorname{Re}(A x, x) \\
\Rightarrow \quad\|x\|^{2} & =2 \lambda \operatorname{Re}(A x, x) \\
\Rightarrow \quad 1 \quad & =2 \lambda \operatorname{Re}(A y, y) \text { where } y=\frac{x}{\|x\|} \\
& =\operatorname{Re}(A y, y)=\frac{1}{2 \lambda} \text { for all } y \in H
\end{aligned}
$$

We have

$$
\begin{aligned}
2 a & =z+\bar{z} \text { i.e. } \quad a=\frac{1}{2}(z+\bar{z}) \\
\text { i.e. } \quad \operatorname{Re}(z) & =\frac{1}{2}(z+\bar{z})
\end{aligned}
$$




$$
\begin{aligned}
& =\frac{1}{2}((A y, y)+(\overline{A y, y})) \\
& =\frac{1}{2}\left((A y, y)+\left(\overline{y, A^{*} y}\right)\right) \\
& =\frac{1}{2}\left((A y, y)+\left(A^{*} y, y\right)\right) \\
& =\frac{1}{2}\left(\left(A+A^{*}\right) y, y\right) \\
& =\left(\frac{1}{2}\left(A+A^{*}\right) y, y\right) \\
& =(\operatorname{Re}(A) y, y)
\end{aligned}
$$

Hence from (8) and (9) we have

$$
\begin{aligned}
& ((\operatorname{Re} A) y, y)=\frac{1}{2 \lambda} \text { for all } y \in H \\
\Rightarrow \quad & W(\operatorname{Re} A) \subseteq R /\{0\}
\end{aligned}
$$

\section{References}

[1]. Simmons, G.F.: Introduction to topology and modern analysis. McGraw-Hill, New York (1963)

[2]. Rudin, Walter: Functional analysis (1981).

[3]. Duggal B.P. and Khalagai J.M.: On operator equation $\mathrm{AB}+\mathrm{BA}^{*}=\mathrm{A}^{*} \mathrm{~B}+\mathrm{BA}=\mathrm{I}$, Indian J. Pure Appli. Math., 13(H), 1376-1383 November 1983.

[4]. Duggal B.P. and Khalagai J.M.: On operator equation AB + B $A=A{ }^{*} B+B A=I$, Math Japan, 26(1981)

[5]. R. Nakamato: On the operator equation THT = K Math. Japan; 18 (1973), PP. 251-252.

[6]. G.K. Pederson, M. Takesaki: The operator equation THT = K Proc. Amer. Math. Soc., 36 (1972), PP. 311-312.

[7]. T. Kato: Perturbation theory for linear operator, $2^{\text {nd }}$ Edition, Springer, 1980.

[8]. Taylor, A.E.: Introduction to functional analysis, Wiley, New York, 1958.

[9]. Md. N. Hoda and Mohammad A. Ansari: Some normal solution on operator equation, AIJRSTEM (USA); 16(1); Sep. - Nov. (2016); PP. 49-51.

[10]. R. Meise, D. Vogt: Introduction to functional analysis, Oxford G.T.M.2, Oxford University Press 1997.

[11]. M.H. Mortad: On some product of two unbounded self adjoint operator, Integral Equation Operator Theory 64 (2009), 399-408.

[12]. M.H. Mortad: On Normality of the solution two Normal operators, complex Analysis Oper. Theory, 61 (2012) 105-112, DOI: 10.1007/s11785-010-0072-7.

[13]. M.H. Mortad: Products of unbounded normal operator, (submitted). arXIV: 1202.6143 V1.

[14]. C. Putram: Commutation properties of Hilbert span operators, Springer, 1967.

[15]. W. Rudin: Functional analysis McGraw-Hill, 1991 ( $2^{\text {nd }}$ edition $)$

[16]. Schwanberg, Allen: The operator equation AX - XB = C with normal A and B, Pasifie J. Math., Vol. 102:447-453 (1982).

[17]. Read, Michael and Sincon, Barry: Functional analysis Academic Press, New York (1972).

[18]. Roth, W.E.: The equation AX - YB = C and AX - XB = C in matrices, Proc. Amer. Math. Soc., 5:392-396 (1952).

[19]. Rosen-blum, M.: On operator equation BX - XA = C Duk Math. J., 25, 263-269 (1956).

[20]. Rosen-blum, M.: The operator equation BX $-\mathrm{XA}=\mathrm{Q}$ with self adjoint. A and B. Proc. Amer. Math. Soc., 20:115-120 (1969).

[21]. Bellman, R.: Introduction to matrix analysis, McGraw-Hill, New York (1960).

[22]. Barberian, S.K.: Lectures in functional analysis and operator theory, Springer-Verlag, New York (1979).

[23]. Barberian, S.K.: The numerical range of normal operator, Duke Math. J., 31:479-483. 